\title{
Jetzt die Weichen stellen für ein leistungsfähiges Forschungsdatenzentrum Gesundheit
}

\section{Now is the Time to Establish an Efficient Health Research Data Centre}

\author{
Autoren \\ Enno Swart ${ }^{1}$, Holger Gothe², Falk Hoffmann ${ }^{3}$, Peter Ihle ${ }^{4}$, Sebastian Claudius Semler ${ }^{5}$, Stefanie March ${ }^{6}$
}

\section{Institute}

1 Institut für Sozialmedizin und Gesundheitssystemforschung, Otto-von-Guericke-Universität Magdeburg Medizinische Fakultat, Magdeburg, Deutschland

2 IGES Institut GmbH, Berlin, Deutschland

3 Department für Versorgungsforschung, Carl von Ossietzky Universität Oldenburg, Oldenburg, Deutschland

4 PMV forschungsgruppe an der Medizinischen Fakultät und Uniklinik Köln, Universität zu Köln, Medizinische Fakultät, Köln, Deutschland

5 Geschäftsführung, TMF - Technologie- und Methodenplattform für die vernetzte medizinische Forschung e.V., Berlin, Deutschland

6 Fachbereich Soziale Arbeit, Gesundheit und Medien, Hochschule Magdeburg-Stendal, Magdeburg, Deutschland

\section{Schlüsselwörter}

Forschungsdatenzentrum, Versorgungsforschung, Sekundärdaten, Sozialdaten

\section{Key words}

health services research, health research data centre, claims data, administrative data

online publiziert $\quad 25.10 .2021$

\section{Bibliografie}

Gesundheitswesen 2021; 83 (Suppl. 2): S139-S141

DOI 10.1055/a-1537-9722

ISSN 0949-7013

(c) 2021. Thieme. All rights reserved.

Georg Thieme Verlag KG, Rüdigerstraße 14,

70469 Stuttgart, Germany

\section{Korrespondenzadresse}

Enno Swart

Institut für Sozialmedizin und Gesundheitssystemforschung Otto-von-Guericke-Universität Magdeburg, Medizinische

Fakultät

Leipziger Straße 44

39120 Magdeburg

Deutschland

enno.swart@med.ovgu.de

\section{ZUSAMMENFASSUNG}

Auf der Grundlage der $\S \S 303 a$ bis $303 f$ SGBV werden aktuell die Weichen für eine innovative und leistungsstarke Informations- und Datenplattform gestellt. Dies erfordert die aktuelle und kurzfristige Bereitstellung maßgeblicher Daten des Gesundheitswesens, vor allem von Prozessdaten der gesetzlichen Krankenversicherung und anderer Sozialversicherungsträger, unter Anwendung wissenschaftlich anerkannter Standards und unter gleichzeitiger Einhaltung datenschutzrechtlicher Bestimmungen. In zehn Empfehlungen werden aus der Sicht künftiger Nutzer*innen Erwartungen an ein „ideales“ FDZ Gesundheit gestellt. Der Beitrag richtet sich als Angebot der Wissenschaft und Versorgungsforschung an Entscheidungsträger im Bereich der Politik und der Selbstverwaltung im deutschen Gesundheitssystem, diese bei Aufbau und Weiterentwicklung des FDZ zu unterstützen und kontinuierlich zu begleiten.

\begin{abstract}
Based on sections 303 a to $303 \mathrm{f}$ social code book $\mathrm{V}$, the course is currently being set for an innovative and high-performance national information and data platform. This requires shortterm provision of current and relevant data on the health care system, especially process data on statutory health insurance and other social insurance carriers, using established methodological standards as well as taking into account data protection regulations. From the point of view of future users, expectations regarding an "ideal" research health data centre are formulated in ten recommendations. The present article is an offer from health services researchers intended to support decision-makers in the field of politics and self-administration in the German health system in their task of establishing and further development of a research health data centre.
\end{abstract}


Mit dem Aufbau eines Forschungsdatenzentrums nach den $\S \S 303 a$ bis $303 f$ Sozialgesetzbuch $V$ sowie der Datentransparenzverordnung (DaTraV) werden aktuell die Weichen für eine innovative und leistungsstarke Informations- und Datenplattform gestellt. Dabei ist das übergeordnete Ziel die Verbesserung der gesundheitlichen Versorgung der Bevölkerung. Um dieses Ziel zu erreichen, müssen die erforderlichen Daten bereitgestellt sowie aktuell und kurzfristig unter Anwendung wissenschaftlich anerkannter Standards und unter gleichzeitiger Einhaltung datenschutzrechtlicher Bestimmungen auswertbar sein. Diese Herausforderung kann nur gemeinsam bewältigt werden. Daher richtet sich der vorliegende Beitrag auch an Entscheidungsträger im Bereich der Politik und der Selbstverwaltung im deutschen Gesundheitssystem und ist als Angebot der Autorengruppe zu verstehen, den Aufbau zu unterstützen und zu begleiten. Der Aufbau des Forschungsdatenzentrums (FDZ) als Weiterentwicklung des Informationssystems Versorgungsdaten macht es möglich, visionär zu denken. Diese Chance nehmen wir als Versorgungsforscher*innen wahr und beschreiben in zehn Empfehlungen die Anforderungen an ein “ideales” FDZ aus der Sicht der Nutzer*innen. Diese Empfehlungen konkretisieren damit allgemein gehaltene Ausführungen aus dem im März 2021 vorgelegten SVRGutachten zur "Digitalisierung für Gesundheit - Ziele und Rahmenbedingungen eines dynamisch lernenden Gesundheitssystems".

Dieses Diskussionspapier wurde erstellt durch Vertreter der Arbeitsgruppe Erhebung und Nutzung von Sekundärdaten (AGENS) der Deutschen Gesellschaft für Sozialmedizin und Prävention (DGSMP) und der Deutschen Gesellschaft für Epidemiologie (DGEpi), der Arbeitsgruppe Validierung und Linkage von Sekundärdaten des Deutschen Netzwerks Versorgungsforschung (DNVF) und der TMF - Technologie- und Methodenplattform für die vernetzte medizinische Forschung e.V.

\section{Empfehlung 1: Das Datenangebot fokussiert auf umfassende Gesundheitsdaten}

Die Routinedaten der gesetzlichen Krankenversicherung (GKV) bilden die grundlegende Datenbasis für das FDZ, indem sie neben den Stammdaten auch das medizinische Versorgungeschehen sektorübergreifend und umfassend abbilden. Mittelfristig sind möglichst alle gesundheitsrelevanten Daten der Kranken- und Pflegeversicherung und weiterer Datenhalter zu integrieren und zeitnah im Längsschnitt verfügbar zu machen.

\section{Empfehlung 2: Durch Datenlinkage kann das Spektrum erweitert werden}

Neben der individuellen Verknüpfung der im FDZ vorgehaltenen Daten untereinander ist die Verlinkung (Datenlinkage) mit externen Daten anzustreben, z. B. Datenlinkage von Primärstudiendaten und Daten anderer Sozialversicherungsträger bzw. FDZs, Registern, aber auch Datenlinkage mit neuen innovativen Datenstrukturen wie beispielsweise der elektronischen Patientenakte oder Daten der Medizininformatik-Initiative. Somit wird auch der Weg zu einer wechselseitigen Validierung der Datenkörper geebnet. Zudem wird vermieden, dass jedes Projekt eigene Infrastrukturen ressourcenintensiv aufbauen muss.

\section{Empfehlung 3: Datentreuhandschaft schützt sensible Gesundheitsdaten}

Gesundheitsdaten als sensible Daten unterliegen nach EU-Recht einer besonderen Schutzwürdigkeit. Das FDZ gewährleistet mit rechtlichen, technischen und organisatorischen Mitteln die Einhaltung aller datenschutzrechtlichen und ethischen Vorgaben und verhindert und ahndet Missbrauch. Bei der Ausübung der Datentreuhandschaft sind mögliche Interessenskonflikte des FDZ zu berücksichtigen: Das FDZ steht in der Datennutzung nachweislich außerhalb jeglichen wirtschaftlichen und wissenschaftlichen Wettbewerbs und übt die Datentreuhänderschaft unparteilich aus.

\section{Empfehlung 4: Datenzugang und Datenzugriff sind forschungsfreundlich}

Die im FDZ vorgehaltenen Daten entfalten ihr Potenzial nur dann, wenn die Daten schnellstmöglich zur Generierung von Forschungsergebnissen bereitgestellt werden und damit die Aktualität der Daten gewährleistet ist. Eine umfassende Dokumentation der verfügbaren Datenkörper hinsichtlich ihres Ursprungs und ihrer Inhalte ist für die Arbeit unerlässlich. Der Zugriff auf projektspezifische Daten im Sinne von Testdaten muss notwendige iterative Plausibilisierungs- und Auswertungszyklen ermöglichen. Das damit verfolgte Ziel ist es, interaktiv syntaktisch korrekte, inhaltlich geprüfte und ausführbare Auswertungsprogramme zu entwickeln, beispielsweise durch personenbezogene sichere Datenfernzugriffe.

\section{Empfehlung 5: Transparente Prozesse sichern die Zielerreichung}

Die Ausgestaltung der Verfahrensregeln ist bei gleichzeitiger Wahrung datenschutzrechtlicher Vorgaben am bevölkerungsbezogenen Nutzen ausgerichtet. Kriterien für die Antragsbewilligung müssen transparent und nachvollziehbar sein, ebenso wie alle weiteren Schritte der Antragsbearbeitung inklusive zeitlicher Vorgaben im Sinne der Planungssicherheit. Im Rahmen der Ergebnisprüfung sind auch das Forschungsinteresse und der damit verbundene mögliche Nutzen in Bezug auf die Verbesserung der Gesundheitsversorgung für Bürger* innen zu berücksichtigen. Die Verantwortung für die Richtigkeit der Analyseergebnisse liegt ausschließlich in der Hand der Forschenden. Die Arbeitsweise und Zielsetzung des FDZ muss transparent gegenüber den primär Nutzenden der Daten dargelegt werden.

\section{Empfehlung 6: Beratung fördert die Datennutzung}

Das FDZ betreibt eine Informationsplattform, auf der den Datennutzenden alle Informationen im Rahmen des Datenanforderungsprozesses umfassend, detailliert und kommentiert zur Verfügung gestellt werden. Das FDZ berät die Forschenden bei Bedarf. Regelmäßige Basisauswertungen oder Methodensammlungen unterstützen den interaktiven Forschungsprozess. Das FDZ versteht sich hierbei als lernendes System im Sinne einer stetigen Weiterentwicklung der Qualität der bereitgestellten Daten. Hierfür notwen- 
dige eigene Methodenforschung mit einem eigens dafür bereitgestellten Budget sind dem FDZ zu ermöglichen.

\section{Empfehlung 7: Das FDZ sucht aktiv die Bürgernähe}

Das FDZ ist bzgl. seiner Ziele, Prozesse und Projekte offen und transparent. Dies betrifft auch die Transparenz gegenüber allen Bürger* innen. Das FDZ soll die breite Öffentlichkeit aktiv und kontinuierlich ansprechen. Über die Arbeit des FDZ und mit seinen Daten generierte Forschungserkenntnisse ist auf dessen Webseite und in anderen geeigneten Medien zu berichten.

\section{Empfehlung 8: Das FDZ arbeitet rechtssicher}

Für den Betrieb des FDZ existiert eine handlungssichernde Rechtsgrundlage. Das FDZ erhält damit einen Handlungsrahmen, innerhalb dessen die zeitnahe Bereitstellung der Daten und schnelle Bearbeitung der Anträge ermöglicht wird. Regelmäßige Evaluationen unter Einbeziehung der Erfahrungen der Nutzer* innen und sich daraus ableitbare Nachbesserungen im Hinblick auf Struktur und Arbeitsprozesse des FDZ gewährleisten einen notwendigen Qualitätsstandard auch im Hinblick auf die bereitgestellten Daten.

\section{Empfehlung 9: Das FDZ arbeitet auf sicherer Finanzierungsgrundlage}

Im Rahmen seines gesetzlichen Auftrags agiert das FDZ effizient auf Basis einer soliden Grundfinanzierung. Grundsätzliches Ziel muss sein, die Datennutzung zu realistischen Kostenkonditionen beantragen zu können, damit dringend notwendige Versorgungsforschung möglich ist. Eine Mischfinanzierung mit Einsatz von Steuermitteln oder Versichertenbeiträgen für den Basisbetrieb des FDZ sowie aufwandsabhängige Gebühren und pauschalierte Fördermittel sind anzustreben.

\section{Empfehlung 10: Nutzervertretung und wissenschaftlicher Beirat sichern eine nachhaltige Entwicklung}

Der nach §303d (2) SGB V vorgesehene „Arbeitskreis der Nutzungsberechtigten “ wirkt in einem partizipativen Prozess beratend an der „Ausgestaltung, Weiterentwicklung und Evaluation des Datenzugangs “ und der Arbeit des FDZ mit. Über den Arbeitskreis werden auch die Aufgaben eines wissenschaftlichen Beirats sichergestellt, der die Arbeit des FDZ wissenschaftlich unterstützt, ebenso eine kontinuierliche Einbindung der Perspektive der Bürger*innen als letztliche Nutznießer*innen der Forschung. Die Einbeziehung der Forschenden und der Patient* innen sowie Bürger* innen sichert eine nachhaltige und öffentlich akzeptierte Weiterentwicklung des FDZ. BMG und BfArM sichern die Finanzierung dieser wichtigen flankierenden Maßnahmen.

\section{Wie geht es nun weiter?}

Manche der aufgezeigten Statements sind in der Gesetzesgrundlage und der Neufassung der Datentransparenzverordnung bereits verankert. Die Rechtssicherheit ist dadurch per se gegeben. Die be- reitzustellenden Daten sind detailliert beschrieben und besitzen den für die Beurteilung des Versorgungsgeschehens im Bereich der gesetzlichen Krankenversicherung notwendigen Detaillierungsgrad. Die Möglichkeit des Datenlinkage, hier sei stellvertretend die elektronische Patientenakte genannt, erweitert die Auswertungsoptionen enorm und sollte gezielt ausgebaut werden. Die Einbeziehung der Daten der Sozialen Pflegeversicherung ist mittelfristig anzustreben.

Die größte Herausforderung liegt aktuell in der datenschutzrechtlich konformen Bereitstellung der sensiblen Gesundheitsdaten mit Wahrung des Identitätsschutzes der Betroffenen. Diese Datenbereitstellung muss gleichzeitig nutzer*innenfreundlich gestaltet werden, um das erklärte Ziel der Verbesserung der Gesundheitsversorgung zu erreichen, die wiederum den Betroffenen - hier den Versicherten - zu Gute kommt. Hierfür gibt es bereits praxisbezogene Lösungsansätze. Das Forschungsdatenzentrum des Statistischen Bundesamtes, das vom Rat für Sozial- und Wirtschaftsdaten akkreditiert ist, stellt verschiedene Datenbestände für wissenschaftliche Auswertungen unter Anwendung einheitlicher und transparenter Verfahrensregelungen zur Verfügung. Das FDZ der Bundesagentur für Arbeit (BA) im Institut für Arbeitsmarkt- und Berufsforschung (IAB) ermöglicht es externen Wissenschaftler* innen, Zugänge zu Mikrodaten für die Forschung im Bereich der Sozialversicherung und der Arbeitsmarkt- und Berufsforschung zu nutzen. Generische Datenschutzkonzepte der TMF können hier leitend sein, ebenso wurden Entwürfe für tragfähige Treuhandkonzepte von der Stiftung Neue Verantwortung beschrieben. Die Gute Praxis Sekundärdatenanalyse und die Gute Praxis Datenlinkage ergänzen diese Modelle und Konzepte aus der konkreten Sicht der Nutzer*innen von Sekundärdaten, hier insbesondere von GKVRoutinedaten. Jetzt gilt es, gemeinsam die Anwendungsfälle zu definieren und problemadäquate Lösungen zu finden. Jetzt werden die Weichen gestellt.

The Time Is Now!

\section{Danksagung}

Wir danken für die inhaltliche Zuarbeit und Diskussion: Silke Andrich und Andrea Icks (Institut für Versorgungsforschung und Gesundheitsökonomie, Medizinische Fakultät und Universitätsklinikum Düsseldorf), Ingo Meyer (PMV forschungsgruppe, Universitätsklinikum Köln), Ulrike Nimptsch (Fachgebiet Management im Gesundheitswesen, Technische Universität Berlin), Nadine Scholten (Institut für Medizinsoziologie, Versorgungsforschung und Rehabilitationswissenschaft, Humanwissenschaftliche Fakultät und Medizinische Fakultät, Universität zu Köln), Mandy Schulz (Zentralinstitut für die kassenärztliche Versorgung in Deutschland, Fachbereich Data Science und Versorgungsanalysen, Berlin), Christoph Stallmann (Institut für Sozialmedizin und Gesundheitssystemforschung, Medizinische Fakultät, Otto-von-Guericke-Universität Magdeburg).

Interessenkonflikt

Die Autorinnen/Autoren geben an, dass kein Interessenkonflikt besteht. 


\section{Hinweis}

Dieser Artikel wurde gemäß des Erratums in der OnlineVersion am 7. Dezember 2021 und am 15. November 2021 korrigiert.

\section{Erratum}

Jetzt die Weichen stellen für ein leistungsfähiges Forschungsdatenzentrum Gesundheit

Swart E., Gothe H, Hoffmann F et al. Gesundheitswesen 2021; doi 10.1055/a-1537-9722

Im oben genannten Artikel wurden die Institutsangaben von H. Gothe ergänzt. Richtig ist:

- Bereich Versorgungsforschung, IGES Institut GmbH, Berlin, Deutschland

- Department für Public Health, Versorgungsforschung und Health Technology Assessment, UMIT - Private Universität für Gesundheitswissenschaften, Medizinische Informatik und Technik GmbH, Hall in Tirol, Austria

- Lehrstuhl Gesundheitswissenschaften/Public Health, Medizinische Fakultät „Carl Gustav Carus“, Technische Universität Dresden, Dresden, Deutschland

Dies wurde in der Online-Version am 15. November 2021 korrigiert.

Im oben genannten Artikel wurde die Institutsangabe von P. Ihle korrigiert. Richtig ist:

PMV forschungsgruppe an der Medizinischen Fakultät und Uniklinik Köln, Universität zu Köln, Medizinische Fakultät, Köln, Deutschland

Dies wurde in der Online-Version am 7. Dezember 2021 korrigiert. 